\title{
Viticulteurs et techniciens viticoles face à leur exposition aux pesticides
}

Technicians and wine growers facing pesticide exposure

\section{Christian Nicourt et Jean-Max Girault}

\section{(2) OpenEdition}

\section{Journals}

Édition électronique

URL : http://journals.openedition.org/economierurale/3789

DOI : 10.4000/economierurale.3789

ISSN : 2105-2581

Éditeur

Société Française d'Économie Rurale (SFER)

Édition imprimée

Date de publication : 15 février 2013

Pagination : 11-25

ISSN : 0013-0559

\section{Référence électronique}

Christian Nicourt et Jean-Max Girault, « Viticulteurs et techniciens viticoles face à leur exposition aux pesticides », Économie rurale [En ligne], 333 | janvier-février 2013, mis en ligne le 15 février 2015, consulté le 19 avril 2019. URL : http://journals.openedition.org/economierurale/3789; DOI : 10.4000/ economierurale.3789 


\section{Viticulteurs et techniciens viticoles face à leur exposition aux pesticides}

Christian NICOURT, Jean-Max GIRAULT • INRA, SAE2, UR 1216 RiTME

nicourt@ivry.inra.fr, girault@ivry.inra.fr

Pourquoi des techniciens viticoles, moins exposés professionnellement aux pesticides que les viticulteurs, mettent-ils en place des stratégies collectives de protection, tandis que ces derniers, à première vue, ne semblent pas en avoir ? Nous soutiendrons l'hypothèse que tous cherchent à limiter une exposition sanitaire qu'ils ne peuvent exclure. L'objet de ce texte vise à comprendre, comparer et décrire leurs stratégies prudentielles.

MOTS-CLÉS : Pesticides, Travail, Métier, Viticulture

\section{Technicians and wine growers facing pesticide exposure}

Why wine technicians, professionally less exposed to pesticides than wine growers, set up collective strategies of protection, while the laters, at first glance, do not seem to have some ? We will support the assumption that both try to limit an exposure they cannot exclude. The object of this text aims to understand, compare and describe their prudential strategies. (JEL: J 29)

KEY-WORDS: Pesticides, Work, Profession, Wine-growing

$\mathbf{E}^{\mathrm{n}}$ Languedoc-Roussillon, où se situe le plus important vignoble de France, l'histoire sociale et sa mémoire ont modelé le travail des viticulteurs au travers du mouvement coopératif. Ce n'est que depuis les toutes dernières années que les caves coopératives n'y sont plus l'organisateur social et politique de chaque commune. Pourtant, en 2005 encore, on compte 314 coopératives qui rassemblent près de 40000 sociétaires cultivant $70 \%$ du vignoble, tandis qu'à peine plus de 5000 vignerons se déclarent en caves particulières et mettent en valeur près de 100000 hectares $^{1}$. Quand les coopérateurs représentent $90 \%$ de la population viticole (hors salariés agricoles plein temps ou saisonniers), le travail viticole prend une forme singulière. Surtout, en s'organisant par commune, la coopération a renforcé les identités locales et plus particulièrement différencié les manières

1. Par comparaison, le second vignoble français, celui d'Aquitaine, compte un peu plus de 11000 exploitations (dont 9000 pour le bordelais) pour une surface de 156000 ha (dont 128000 pour le bordelais). de travailler. Dès lors, une identité de viticulteurs languedociens, liée à une histoire longue, marquée par la révolte emblématique des vignerons en 1907 (Cadé, 1994), cohabite avec celles de viticulteurs dont le travail est modelé autant par leurs terroirs viticoles que par le territoire de leurs caves. Ainsi, les manières de penser, de dire et de faire le travail, consolidées par un répertoire commun d'histoires, d'épreuves et d'expériences peuvent aussi bien rassembler que distinguer les viticulteurs en autant de communautés de pratiques (Wenger, 1998). Ces communautés sont confrontées depuis plusieurs décennies à un usage des pesticides dont les effets sanitairement problématiques sont de plus en plus mis en évidence, aussi bien par des procédures contentieuses que par les déclarations des plus hautes autorités de l'État. Ces procédures et ces déclarations ne semblent pas avoir sensiblement affecté les manières de travailler des viticulteurs ni modifié ce qu'ils considèrent comme une normalité sanitaire (Canguilhem, 1966): des maux liés aux traitements, dont les manifestations ne méritent pas leur attention et leur 
intervention. Mais alors, pourquoi des techniciens viticoles, moins exposés professionnellement aux pesticides que les viticulteurs, mettent-ils en place des stratégies collectives prudentielles (Cru et Dejours, 1983), tandis que ces derniers ne semblent pas en avoir? Notre hypothèse est que tous inventent des manières de faire pour limiter une exposition sanitaire qu'ils ne peuvent exclure. Dès lors, ce sont les expériences de leur travail et ce qu'ils considèrent comme une normalité sanitaire admises par leurs communautés de pratiques voire leurs cultures professionnelles, qui orientent la normalisation de leur usage des pesticides.

L'objectif de notre propos est de comprendre les stratégies prudentielles de ceux qui sont exposés professionnellement aux pesticides dans leurs situations de travail. Plus particulièrement, il s'agit de comprendre, au niveau de leurs communautés de pratiques et de leurs cultures professionnelles, ce qui les conduit à construire et à admettre des normes communes et distinctes dans des contextes à risque communs. Pour cela, nous avons testé notre hypothèse en comparant deux populations: des viticulteurs et des techniciens viticoles. Ces deux populations sont liées entre elles, notamment par un problème sanitaire commun, bien qu'elles subissent des intensités et des durées d'exposition sans commune mesure. De prime abord, les techniciens ne semblent pourtant pas concernés. Ils assument en effet le triptyque des fonctions du conseil agricole mis en évidence par Compagnone et Cerf (2000). La fonction prescriptive fournit des informations destinées à orienter le choix des viticulteurs pour traiter, tandis que la fonction de résolution de problèmes répond à des demandes singulières des viticulteurs et celle de pilotage implique que le conseiller assume la responsabilité de ses conseils. Les conseillers assurent ces fonctions aussi bien dans des cadres collectifs (réunion à la coopérative pour expliciter la mise en place d'un nouveau cahier des charges, opération de contrôle et d'amélioration des performances des pulvérisateurs dans une petite région) que plus individualisés (permanence de conseil à la cave coopérative ou à l'échelon local de la Chambre d'agriculture...). Dans ces contextes, ils sont jugés sur leur capacité à orienter les manières de faire des viticulteurs vers de «bonnes pratiques », mais ils doivent se faire « transparents » : les résultats issus de leur conseil sont ceux des agriculteurs (Brives, 2006). C'est aussi parce que leur conseil vise à faire s'interroger les viticulteurs sur des hypothèses de résolutions techniques plutôt que - comme les technico-commerciaux des entreprises privées autant que des coopératives d'approvisionnement - leur proposer des recettes (Compagnone, 2006). Mais les techniciens sont aussi dans les vignes, pour évaluer les situations et nourrir leurs conseils. Et pour cela, «il y a un temps d'exposition, et on touche beaucoup! » (Débat techniciens, CRA Languedoc-Roussillon).

Notre $\operatorname{propos}^{2}$ s'appuie sur des entretiens $^{3}$ conduits de 2005 à 2007, auprès de 51 viticulteurs coopérateurs du Languedoc-Roussillon, et de 19 conseillers techniques viticoles de caves coopératives et de Chambres d'agriculture $(\mathrm{CA})^{4}$.

2. Les résultats exposés sont issus d'un rapport de recherche pour le Programme «Évaluation et réduction des risques liés à l'utilisation des pesticides » du ministère de l'Écologie et du Développement Durable (Nicourt et al., 2007).

3.Les entretiens sont de type semi-directifs: orientés par un canevas d'entretien qui laisse libre cours aux sujets que veulent aborder les enquêtés. Ils sont enregistrés puis retranscrits pour constituer le matériau de l'analyse.

4. Nous avons également assisté à neuf réunions de viticulteurs dans des caves coopératives et nous avons observé une situation de débat entre viticulteurs à propos de l'usage des pesticides : un contrôle technique public de pulvérisateurs organisé par une CA, impliquant une douzaine de viticulteurs et quatre conseillers viticoles. 
Les viticulteurs ont des situations modestes comparées à ceux d'autres vignobles plus prestigieux (Bourgogne et Bordeaux notamment) et, en Languedoc-Roussillon, d'autant plus qu'ils ne sont pas en caves particulières. Les techniciens viticoles dépendent des $\mathrm{CA}$ ou des caves coopératives ; contrairement à d'autres - ceux des coopératives d'approvisionnement qui vendent matériels, engrais et pesticides - ils interviennent dans les vignes et conseillent les viticulteurs, dans une perspective de réduction de l'usage des pesticides. Cet usage est une préoccupation commune des deux métiers et ceux qui les exercent sont tous exposés.

Les entretiens avaient pour objectif initial de mettre en évidence l'évolution récente du travail de viticulteurs confrontés aux prescriptions leur enjoignant de réduire l'usage des pesticides. Dans une perspective compréhensive, où il s'agit de saisir le sens que les acteurs donnent à leurs actions (Hughes, 1996), la focalisation de notre questionnement - généralement en dernière partie des entretiens - sur la relation entre cet usage et leurs manières de se protéger des risques encourus, s'est heurtée à un double problème méthodologique. Poser aux acteurs des questions sur des sujets envers lesquels ils adoptent des attitudes de déni, c'est-à-dire dans la perspective de Dejours (2000) d'occultation d'une partie de la réalité de leur travail génératrice de peur ou de souffrance - qui plus est dans le cadre de la violence symbolique d'une situation d'entretien conduit à des discours souvent stéréotypés ou évasifs. Et cette violence est redoublée lorsqu'elle implique la mise au jour de situations douloureuses, souvent refoulées. Dans ce contexte les $2 / 3$ des viticulteurs se sont dérobés à ce questionnement. Il faut cependant noter que les récits les plus étoffés l'ont été lors d'entretiens menés après ou lors de la visite de parcelles, plutôt que lorsque ceux-ci avaient lieu soit chez le viticulteur, soit à la coopérative. Soulignant ainsi la pertinence de l'entretien sur le terrain qui permet au viticulteur autant de se remémorer que de "démontrer» la situation vécue. Par contre, des techniciens ont posé d'emblée, lors d'une réunion sur le terrain, le problème de leur propre exposition aux pesticides lors de l'exercice de leur métier. De même que les viticulteurs, certains d'entre eux ont ensuite esquivé ce questionnement. Celui-ci a visé alors à caractériser leurs situations d'exposition aux pesticides et à les interroger sur leurs manières de les gérer, notamment comme problème de leur métier.

Dans ce texte, nous décrivons d'abord comment l'usage des pesticides est devenu une préoccupation commune des viticulteurs et des techniciens. Pour ceux qui sont exposés à leurs épandages, c'est l'appréciation de la normalité sanitaire acceptable au travail qui a changé. Ce qui a conduit certains à se préoccuper de leur protection et à en appréhender les limites : une défiance partagée envers les dispositifs de protection. Nous examinons ensuite comment les contraintes et les expériences, qui sont apparues lors des épreuves affrontées par chacun pour se protéger, conduisent à une obligation de normativité, c'est-à-dire, dans la perspective de Canguilhem (1966) à créer de nouvelles normes et à les adapter pour répondre à l'évolution des situations. Face à ce qui se conçoit désormais comme un risque sanitaire, il faut construire, admettre et partager de nouvelles normes, soulignées par des écarts, qui traduisent les relations entretenues par les viticulteurs au sein de communautés de pratiques ancrées dans des territoires. Tandis que pour les techniciens, cette normativité met surtout en avant une distinction entre femmes et hommes dans les manières de se protéger. Dès lors, nous interprétons les similitudes et les divergences des réponses des techniciens et des viticulteurs comme l'expression différenciée de cultures de métier, qui 
s'expriment sous la forme de manières de penser et d'exercer le travail, selon un système de valeurs.

\section{Quelle normalité sanitaire de l'exposition aux pesticides ?}

La normalité sanitaire au travail est le fruit d'épreuves renouvelées, dont la dimension éprouvante des maux s'est érodée, pour devenir banalité de l'expérience du quotidien du travail. Ces maux, devenus ordinaires, peuvent prendre cependant un autre relief lorsqu'ils sont confrontés à des situations extraordinaires. C'est ainsi que des alertes sanitaires sont susceptibles de remettre en question la banalisation initiale.

\section{Les maux ordinaires d'un travail exposé aux pesticides}

L'exposition des viticulteurs aux pesticides est liée à leurs activités de préparation et d'épandage. Celles-ci sont renouvelées tout au long du cycle végétatif de la vigne et les périodes d'exposition continues sont souvent supérieures à la demi-journée ou à la demi-nuitée. La préparation des produits et l'entretien du matériel, comme les observations et les manipulations dans les vignes traitées, sont d'autres contextes d'exposition. De même, les techniciens viticoles sont exposés lorsqu'ils effectuent des analyses et des prélèvements dans des vignes préalablement traitées; comme ils l'expliquent : "Il y a un temps d'exposition et on touche beaucoup. » À l'occasion des épandages, analyses et prélèvements, viticulteurs et techniciens ressentent des irritations, des céphalées et des nausées, vomissent... De tels maux, parce qu'ils se présentent de manière fugace et peuvent être assignés à d'autres origines, sont le plus souvent minorés, ignorés, oubliés ou déniés. C'est ainsi qu'ils sont considérés comme des attributs ordinaires du travail, au même titre que la fatigue des longues journées ou que la pénibilité physique issue de manipulations.
"Si vous disiez maux de tête, on disait qu'il restait peut-être un peu trop longtemps au soleil et pas particulièrement qu'il inhalait le contenu de la cuve. »

(Viticulteur, Aude, $n^{\circ}$ 16)

« Je crois qu'on est tous pareils. Le soir, on peut être fatigué, avoir mal à la tête, on sait pas si on a mal à la tête parce que la journée a été fatigante ou si c'est un produit qu'on a accumulé. On ne peut pas savoir. »

(Débat techniciens, CRA Languedoc-Roussillon)

Viticulteurs et techniciens relient cependant certains de ces maux à leurs activités professionnelles, même si tous en minimisent la portée. Les uns, en rappelant les railleries qui associent les maux éprouvés à ceux de situations quotidiennes, pour mettre à distance leur peur éventuelle. Les autres en leur attribuant des causes tout aussi quotidiennes. Il s'agit là d'ancrer les situations sanitairement problématiques dans le quotidien, pour qu'elles ne fassent plus saillance et puissent être banalisées ou déniées.

\section{Le partage d'alertes sanitaires}

L'expérience des maux du travail met cependant en évidence des situations préoccupantes, parce qu'emblématiques du risque, c'est-à-dire considérées comme telles dans d'autres contextes sociaux. Ainsi, les émissions d'odeurs sont considérées par les viticulteurs comme révélatrices de risques toxiques. Une telle liaison s'inscrit dans une histoire longue. Pour les hygiénistes déjà, les odeurs «frappent les sens, et produisent sur les organes les moins délicats une impression pénible et repoussante qui se trahit par une répugnance instinctive et semble l'avertissement d'un danger réel » (Tardieu, 1862). Cette liaison, qui tend à être socialement partagée, est d'ailleurs aujourd'hui utilisée pour avertir d'un tel risque : un composé 
soufré, ajouté au gaz domestique, indique le danger d'une fuite à l'usager. De même que dans les vignes, l'odeur perçue par le viticulteur lui signale la perfectibilité de ses éventuelles protections, elle indique au technicien un traitement récent de la parcelle de vigne dans laquelle il intervient.

"L'étanchéité n'est jamais à $100 \%$ dans la cabine. Et à des moments, quand y a pas de vent, ça fait un nuage, un brouillard qui, des fois, vient dedans. L'air qu'on filtre, je suis sûr qu'il est pas pur. Il y a des moments, l'odeur rentre dans la cabine, donc quelque part, le produit rentre aussi.»

(Viticulteur, Aude, $\mathrm{n}^{\circ} 22 \mathrm{~h}$ )

"Dans une parcelle qui sentait où on s'était arrêté, on n'allait pas au milieu de la vigne, mais on va faire quelques observations. Mais là, moi je m'en vais, je remonte dans la voiture, mais avant, non. Mais c'est pas parce que ça sentait mauvais que c'était forcément plus toxique. Mais il y avait l'indicateur : elle a été traitée récemment. »

(Techniciens, CRA Languedoc-Roussillon)

C'est à partir de l'expérience de l'usage des produits que les viticulteurs s'interrogent. Leur appréhension s'adosse à l'expérience de maux endurés ou à celle de collègues. Mais ils sont d'autant plus en alerte que, lorsque l'usage de tels produits leur est imposé, ils en discutent collectivement. Ainsi, des traitements contre la flavescence dorée, rendus obligatoires par arrêtés préfectoraux. Dans ce cas, les débats portent autant sur l'opportunité et l'efficacité de l'obligation que sur ses effets sanitaires. Incertains quant à la portée sanitaire des maux ordinaires, les viticulteurs sont particulièrement diserts à propos de la mortalité de proches, collègues ou parents. Chacun cite des cas de cancer, au point que cette maladie apparaît comme une expérience territoriale du métier.
«Des fois, on en parle et on me dit: Tiens, celui-là est mort du cancer. L'autre fois, j'étais avec un ami, sur une vingtaine de maisons, presque tous avaient eu un cancer, que des agriculteurs. On se dit: quand même. »

(Viticulteur, Pyrénées orientales, $\mathrm{n}^{\circ} 20 \mathrm{~h}$ )

«C'est des produits quand même on sait pas trop... C'est vrai qu'on a mon oncle qui était agriculteur, qui a eu un cancer du cerveau, donc ça vient un petit peu des produits, des trucs comme ça. »

(Couple de viticulteurs, Hérault)

La survenue de pathologies inhabituelles alerte les techniciens. En prenant leur cas ou celui de collègues pour référence, ils montrent la croissance d'une interrogation sur la relation entre leurs problèmes de santé et cette exposition.

"Moi? j'ai un problème aux cordes vocales. J'ai été voir à la Mutualité, la médecine du travail, on n'a pas relié aux produits. »

(Technicien CA, Hérault)

"J'ai une collègue qui a fait faire des analyses de sang en recherchant je sais plus quoi... Et il y avait un lien. »

(Technicien CA, Hérault)

Les situations d'alerte ébranlent périodiquement la normalité sanitaire construite dans les tâches quotidiennes des techniciens comme des viticulteurs.

\section{L'incertitude sanitaire des situations de travail}

Parce que la normalité sanitaire du travail ordinaire des viticulteurs comme des techniciens est mise à l'épreuve par la survenue fréquente de situations d'alerte, certaines tâches deviennent sanitairement incertaines. Pour les viticulteurs, l'incertitude est d'abord celle des effets sanitaires 
de l'épandage des produits. Tandis que la portée des maux ordinaires pourrait être minorée dans le quotidien, leur répétition sur la durée interroge.

"C'est pas une intoxication aiguë qui me fait peur, moi ? c'est sur 40 ans de carrière. C'est plus ça qui me fait réfléchir. »

(Viticulteur, Hérault, en GAEC)

Pour les techniciens, l'incertitude est celle des traitements effectués au préalable lorsqu'ils interviennent sur une parcelle. Ils ne peuvent savoir s'ils s'exposent sanitairement lorsqu'ils effectuent un prélèvement, à moins qu'une odeur leur signale un traitement récent mais certains pesticides sont inodores.

«On rentre dans les vignes, on ne sait pas quand elles ont été traitées, sauf si ça pue vraiment... Il y a des fois, on rentre (dans les vignes) et puis il avait dûfaire un passage la veille, alors on a mal à la tête et le lendemain c'est passé. »

(Technicien CA, Hérault)

Viticulteurs et techniciens partagent une même défiance envers les dispositifs de protection, d'abord parce que ceux-ci sont inappropriés aux conditions réelles d'un travail impliquant une variabilité de tâches et de contextes. Les combinaisons sont de véritables étuves par grande chaleur, les gants limitent la finesse des manipulations, les masques ne sont pas plus aisés à porter qu'à entretenir... Pour les viticulteurs, les cabines filtrantes et pressurisées sont mal adaptées aux contraintes techniques du travail : obligations d'effectuer des réglages extérieurs ou d'utiliser des commandes qui impliquent des ouvertures de cabines, projection de brouillard sur les vitres, sans compter la nécessité de changer fréquemment les cartouches... Ils doutent de l'efficacité des protections prescrites, même s'ils les utilisent. C'est aussi ce qui explique l'hétérogénéité de leurs manières de se protéger, au-delà même de la variété des contextes et des produits épandus ; au-delà aussi d'un compromis entre le coût financier, la protection espérée et les contraintes subies. D'autant que la permanence des maux, ou la présence de l'odeur des produits, leur a appris que l'efficacité des protections était perfectible. Les techniciens sont semblablement dubitatifs.

«Je mets un masque, j'ai des lunettes, donc je mets le masque, je mets la capuche, je mets des gants. Je mets une combinaison jetable. Mais je m'aperçois que, des fois, j'ai un peu mal au ventre ou je suis un peu barbouillé mais sans plus, malgré tout ça... Traiter avec des capuches, le masque. Quand vous passez quatre heures comme ça, c'est pas idéal. »

(Viticulteur, Hérault, n 20-58)

"Quand on fait les observations, on a des gants maintenant, un grand masque. Je ne sais pas si je le mettrai encore cette année. »

(Technicien CA, Hérault)

Les travaux de Garrigou et al. (2008), révélant une contamination plus forte de ceux qui portent des combinaisons lors de certains travaux, confortent les doutes des viticulteurs. Ils montrent que cette contamination peut renvoyer autant à des pratiques de moindre vigilance liées à des « croyances de surprotection », qu'à des problèmes d'entretien voire de qualité des équipements de protection. Tandis que des essais ont démontré la perméabilité aux pesticides de combinaisons certifiées (Garrigou, 2007), la protection assurée par les cabines semble aussi perfectible. D'autant que, si les traitements varient selon les exploitations, les durées d'exposition sont toujours conséquentes.

"On essaye de s'empoisonner le moins possible et c'est difficile. Moi, on est trois sur mon exploitation: mon père et moi et mon beau-frère. On traite quand même, enfin moi je traite 25 hectares tous les quinze jours. Ça me fait normalement deux 
jours, enfin deux nuits de traitements, deux fois 12 heures. Bon, on a les cabines avec les filtres, on les change. On fait tout ce qu'il faut mais c'est pas assez... Donc ça a un impact quand même, parce qu'on passe un temps monstrueux à ça. »

(Viticulteur, Hérault, $n^{\circ} 45$ )

Les maux banalisés dans le quotidien des situations de travail font saillance lors des alertes : ils pointent l'incertitude sanitaire. Cette saillance interroge et conduit à faire évoluer les manières de faire.

\section{L'obligation de normativité face au risque sanitaire}

Face à l'incertitude sanitaire de leurs situations de travail, les viticulteurs et les techniciens inventent des manières de faire prudentielles distinctives. Les viticulteurs rusent individuellement avec le danger tout en intégrant les pressions de la rurbanisation. Tandis que les techniciens cherchent à éviter d'être exposés. Ces pratiques prudentielles sont revendiquées comme telles. Elles rompent avec les usages construits et reconnus dans leurs communautés de pratiques. En ce sens, elles interpellent les manières de faire des métiers.

\section{Adapter les pratiques à la rurbanisation, pallier les carences des dispositifs de protection}

Comme l'ensemble des agriculteurs, les viticulteurs sont confrontés à une recomposition de l'espace rural, qui passe par une modification tant de ses populations que de l'organisation de l'espace, et un changement de son sens (Jollivet et Mathieu, 1989). La ruralité devient un décor, envers du monde urbain. Pour les viticulteurs, cela signifie une perte d'influence dans des communes où l'activité de la cave coopérative rythmait la vie sociale. C'est aussi un autre regard porté sur le travail viticole. Entre les viticulteurs et leurs voisins, le bruit des engins et l'épandage des pesticides à proximité des habitations deviennent objets de conflits. Dès lors, ils doivent composer avec leurs nouveaux concitoyens en modifiant leurs manières de travailler.

Il y a cinq ans, il y avait pas d'habitations. La première habitation était à $1,5 \mathrm{~km}$ et maintenant il y a le lotissement avec 80 maisons qui sont à $500 \mathrm{~m}$... C'est pour ça qu'il faut faire un maximum gaffe avant de se faire remonter les bretelles: d'aller jouer sur les heures de traitement... J'ai un gendarme... Entre le mur mitoyen et la première souche, il y a 5 mètres... Je suis un peu emmerdé pour traiter... Quand il y a le vent dominant en tramontane, la maison est sous le vent, je suis obligé de couper trois ou quatre mètres avant d'arriver, de tourner, avec le pulvé coupé, ensuite, de reprendre et de faire tous les bords avec les bras que d'un côté, pour finir mon traitement.

(Viticulteur, Pyrénées orientales, $n^{\circ} 20$ )

Quand les viticulteurs deviennent une population minoritaire des communes viticoles, la signification sociale des traitements change. D'opération technique d'un métier partagé par les habitants de la commune, ils deviennent une pratique dangereuse faisant subir un risque toxique à la population locale et à l'environnement. La visibilité publique du travail viticole implique d'en rendre les pratiques tolérables ou, à tout le moins, de ne pas mettre en œuvre de pratiques devenues emblématiques des risques toxiques. En rappelant l'existence du risque, la rurbanisation influence les manières de travailler. Cependant, dans cet espace en voie de « désagricolisation» (Luginbühl, 1991), l'objectif du viticulteur n'est pas de diminuer son exposition au risque toxique, mais à celui des critiques que suscitent ses épandages. Ces critiques ont en effet un coût humain. À l'instar des autres travaux agricoles porteurs de critiques sociales, c'est donc aussi en termes de souffrance 
au travail et d'identité professionnelle qu'il faut considérer l'épandage des pesticides (De Gasparo et al., 2006). Dès lors, certains viticulteurs délaissent les protections visibles qui soulignent les épandages.

«Maintenant les vignes, elles se retrouvent en bordure des maisons. Un agriculteur qui va aller traiter avec une combinaison, masque à gaz et tout ça, il se fait sortir au fusil. »

(Débat techniciens CRA LanguedocRoussillon)

L'épandage des pesticides pose la question de la relation entre santé au travail et santé environnementale au travers des techniques de protection et de leur visibilité publique. D'un côté, les viticulteurs se trouvent confrontés à leurs doutes sur l'efficacité des protections, de l'autre, la visibilité de leur port inquiète les riverains Dès lors, l'inappropriation des protections prescrites aux situations de travail se cumule avec le risque que le viticulteur soit interpellé du fait de leur port. L'usage de techniques de compromis se développe ainsi, chacun feignant d'ignorer que le risque n'a pas varié.

"Quelqu'un qui se promène... Quand il voit un viticulteur avec un masque, il est un peu inquiet quand même... Involontairement, vous donnez un message que vous appliquez un produit qui est nocif ou qui peut présenter une nocivité... Mais quand on est dans une cabine, ça se voit pas. Il vaut mieux avoir une cabine que le masque... La cabine, même si je sais pas si c'est vraiment très efficace, mais bon, la cabine avec les filtres à charbon, je pense que c'est le bon compromis...»

(Technicienne CRA, Languedoc-Roussillon)

Dans un tel contexte, qui impose de subir les risques en déniant les problèmes, les viticulteurs bricolent des manières prudentielles de faire, pour gérer le risque ou le mettre à distance. Cette normativité, surgissant des épreuves endurées, vise à compenser les carences des prescriptions. Faire de l'apnée, plutôt que de se savoir contaminé en utilisant les protections prescrites, peut paraître dérisoire ; cela témoigne aussi d'une reconnaissance et d'une appropriation du risque. Là où son exposition serait maximale, c'est en quelque sorte par ruse que le viticulteur se rend maître du risque en se mettant en apnée. À tout le moins, cette manière de faire est un jeu ironique qui a une efficacité symbolique en lui redonnant une posture d'acteur dans une situation qui le domine (Trompette et Caroly, 2004).

Il y a quelques-uns qui se mettent des combinaisons jetables pour traiter et donc des masques, alors des fois c'est des masques à poussière, ça fait un peu sourire, des fois c'est des masques à cartouche... Moi je préfère encore ne rien mettre parce que dès que vous avez un peu de produit dessus, de suite c'est tout le temps que vous respirez le produit. Moi j'estime, quand on traite, c'est quand on tourne qu'éventuellement on peut en prendre. Eh ben ou on fait l'apnée ou on coupe le pulvé et puis voilà.

(Viticulteur, Pyrénées orientales, $n^{\circ} 45$ )

Entre doutes sur la pertinence des protections et visibilité publique des risques, c'est-à-dire entre santé au travail et santé environnementale, les manières de faire construites par les viticulteurs visent à rassurer chacun : eux-mêmes autant que les riverains.

\section{Les attitudes prudentielles des techniciens}

L'expérience de maux ordinaires ou d'alertes sanitaires conduit les techniciens à se préoccuper de leur exposition aux pesticides. Leur normativité s'oriente davantage vers le retrait des contextes d'exposition plutôt que vers l'usage des protections qui leur sont fournies. Outre l'efficacité 
problématique de ces protections, c'est aussi leur inappropriation au travail qui limite leur usage : contrôles multiples sur des sites différents au cours de la journée impliquant de reprendre un véhicule, se changer... Dans cette perspective, ils cherchent à réduire leur exposition. Ils sont aidés en cela par certains viticulteurs, qui se préoccupent de l'usage des pesticides, parce qu'ils sont insérés dans des groupes de viticulture raisonnée. Ces viticulteurs, sensibles aux risques d'exposition après des épandages, préviennent les techniciens.

"Quand on est avec des groupes de conduite raisonnée, le gars nous dit : "Cet après-midi, il ne faut pas aller dans ma parcelle, je l'ai traitée hier. »Bon, on va en voir une autre."

(Technicien CA, Hérault)

Intervenir auprès de nombreux viticulteurs confronte les techniciens à des postures différenciées d'usage et d'implication sanitaire des pesticides. De cette diversité, ils retiennent les conseils de ceux qui sont en conduite raisonnée. C'est ainsi qu'ils cherchent à étendre à l'ensemble de leurs interventions le principe de ne pas analyser les parcelles récemment traitées. Pour cela, ils envisagent d'interroger préalablement les viticulteurs impliqués, pour qualifier le statut sanitaire des parcelles, et éviter ainsi les vignes contaminées.

"Quand on recherche des parcelles conjoncturelles sur certains parasites, à part l'odeur, on ne sait pas quand le gars a traité. Et c'est pas évident, on en a discuté entre nous: Appeler les gars la veille en demandant quand vous avez traité, si vous allez traiter demain matin, parce qu'on pensait passer. Peut-être qu'il faudra le faire. »

(Technicien CA, Hérault)

Les pratiques prudentielles des techniciens sont assises sur les apports des viticulteurs les plus impliqués dans la gestion précautionneuse des pesticides. Instruits par cette expérience, ils cherchent à développer une attitude de retrait à l'exposition au risque.

Les pratiques prudentielles des viticulteurs sont celles de travailleurs seuls dans les situations à risque. À moins de changer radicalement leurs manières de travailler - passer à la bio - pour eux l'exposition aux pesticides est un « risque du métier », ces maux ordinaires du travail qui appartiennent à l'identité de chaque métier (Moriceau, 2009). La situation des techniciens viticoles diffère. Leur métier s'exerce le plus souvent dans des contextes collectifs, dans lesquels interviennent leurs pairs. Aussi discutent-ils régulièrement entre eux de leurs tâches. De même, travaillent-ils parfois en binômes ou avec les viticulteurs concernés par leurs analyses. Cette dimension collective du travail des techniciens influence notablement leurs pratiques prudentielles. Malgré les différentes modalités que prennent les pratiques prudentielles relatées, elles sont toutes énoncées publiquement comme concourant à la protection de celui qui les met en œuvre. Cette publicité souligne la permanence de la dimension altruiste du travail (Linhart, 2009). Lorsqu'elle s'inscrit dans des collectifs restreints comme dans des communautés de pratiques territorialisées, la normativité se propose comme une contribution à la dynamique du métier.

\section{Réponses aux risques d'exposition aux pesticides comme expressions de cultures de métier}

Les réponses à l'exposition aux pesticides développées par les viticulteurs et les techniciens sont construites à partir de postures professionnelles différenciées. Celle des viticulteurs, travailleurs indépendants - parfois marqués par une rupture générationnelle - encore ancrés dans l'histoire longue du territoire communal, n'est pas 
celle des techniciens et conseillers agricoles, caractérisée par une posture de salariés qui clive femmes et hommes. Dès lors, face à l'exposition aux pesticides, les cultures professionnelles du risque se traduisent par des appropriations distinctes.

\section{Une posture de travailleur indépendant marquée par une rupture générationnelle chez les viticulteurs}

Les viticulteurs du Languedoc-Roussillon ont une relation au risque liée à une histoire qui leur a attribué une identité mêlant celle de l'ouvrier à celle du travailleur indépendant et du coopérateur. Cette construction, qui leur donne une posture ambiguë face au risque, reste d'actualité. En effet, selon les conseillers viticoles, seuls 10 à $20 \%$ des coopérateurs, selon les caves, sont viticulteurs à plein temps. Une majorité est faite de retraités, tandis que 30 à $40 \%$ sont des doubles actifs, le plus souvent ouvriers ou employés. L'identité singulière qui en résulte est remise en cause par les jeunes viticulteurs, plus soucieux de leurs relations au produit et au marché, alors que leurs aînés sont restés plus ancrés dans l'histoire sociale de la coopération et des modèles des manières de bien faire qu'elle véhiculait. Tous assument cependant les risques de leur activité pour assurer leur revenu, les uns dans la perspective ouvrière, les autres dans une perspective entrepreneuriale. Assurer ce revenu passe désormais par une relation plus étroite au marché que tout signal de disqualification influence. Dès lors, il leur faut dénier l'éventuelle contamination du vin, produit de leur travail, qui remettrait en cause sa qualité. Et l'on conçoit que certains veuillent autant occulter l'usage des pratiques à risques, que les maux qui pourraient leur en coûter.

«Avant tu remuais les produits, je te jure, même moi... Tu prenais les gouttes de partout, mais les gens s'en foutaient. Presque à la limite, plus tu en foutais partout, plus tu étais une référence... Mais plus vous traitez, vous arrivez avec le tracteur blanc, bleu, de toutes les couleurs et plus, pour le monde agricole, vous étiez quelqu'un de bien. Et c'est ancré, ça. Ça, c'est un truc qui est encore. Je suis ni vieux, ni jeune, mais les gens qui sont englués dedans, c'est encore ça la référence. »

(Viticulteur, Pyrénées orientales, $n^{\circ} 20$ )

La mise à distance des questions de santé est une banalité du monde du travail, qui a même été magnifiée au travers de la figure de la fatigue dans la population ouvrière (Loriol, 2000). En viticulture, cette culture est fortement représentée par les doubles actifs. Ceux-ci, du fait des exigences temporelles de leur emploi principal, ne traitent généralement que le week-end, c'est-à-dire, selon leurs collègues mono-actifs, «bombardent tous les 14 jours ». Au travers de la stigmatisation des pratiques à risque des doubles actifs, pressés par le temps pour effectuer leurs traitements, ce sont aussi les clivages sociaux du métier qui resurgissent à propos des manières de traiter. De même que les viticulteurs retraités qui cultivent toujours leur vigne, les doubles actifs sont considérés à la marge du métier, d'autant plus que leurs parcelles sont convoitées. Les manières de faire de ces marginaux du métier rendent public ce que les plus jeunes viticulteurs cherchent à occulter. Mais rendre invisible l'usage des pesticides n'est pas à la portée de tous. Dès lors, la diversité des manières de traiter est source de tensions. Elle fragilise la relation au marché dont dépend le revenu de chacun.

"Il y a aussi l'image de marque: Untel, le viticulteur, regarde-le, il est comme un scaphandre, donc il utilise des produits toxiques, donc le vin du coin est un vin traité avec des produits toxiques. Et ça, il y a une image qui est pas trop bonne. »

(Viticulteur, Pyrénées orientales, $n^{\circ} 45$ )

L'identité professionnelle du viticulteur, autrefois essentiellement construite dans la relation aux pairs sur le territoire de la cave 
coopérative, est aujourd'hui influencée par d'autres partenaires : aussi bien les clients qui boivent le vin de la cave, que les riverains impliqués par les traitements phytosanitaires. À ce niveau, la prise accentuée de risque par les viticulteurs s'inscrit dans une forme de négociation entre celui qui le génère et celui qui le supporte : plus la pression rurbaine est forte, plus le viticulteur prend en charge le risque, jusqu'à n'utiliser aucune protection pour en démontrer l'absence. Les manières de traiter sont ainsi influencées par les exigences des populations résidant à proximité des parcelles. Cette volonté que l'usage des pesticides ne puisse être critiqué par les riverains ou par les consommateurs est surtout exprimée par les jeunes viticulteurs. C'est aussi une manière de se démarquer des anciens en rompant avec des manières de faire emblématiques qui avaient été érigées en modèles du bien faire : vignes d'autant plus esthétiques que désherbées, viticulteur d'autant plus vaillant que maculé de «bleu».

«Ils ne savent pas pourquoi ils traitent. Peut-être parce qu'à l'époque le père ou le grand-père disait: il faut traiter tant de fois. Au bout de 14 jours, il faut bombarder. Et puis ça fait pas trop plaisir et je n'arrive pas à comprendre comment des gens peuvent sulfater et peuvent revenir bleu, sur les yeux, partout. »

(Réunion jeunes viticulteurs, Aude)

Chez les viticulteurs coopérateurs, travailleurs indépendants, le déni et la mise à distance de l'exposition aux risques participent d'une culture du métier, renouvelée par une relation forte aux attentes du marché et à celle des populations riveraines.

\section{Chez les techniciens, une posture de salariés, différenciée selon leur genre}

Le travail des techniciens implique une recherche bibliographique permanente, qui les conduit à prendre connaissance des textes concernant la prévention des risques sanitaires liés à l'usage des pesticides. Qu'il s'agisse de la dangerosité des produits, dont ils consultent les fiches techniques indiquant les seuils et les classes de toxicité, ou des textes réglementaires et des travaux scientifiques qui discutent l'exposition professionnelle en ambiances toxiques, les techniciens sont une population qui se documente, d'autant plus avertie qu'elle a eu l'expérience de maux et d'alertes. C'est ainsi qu'ils cherchent à se préserver des risques en quêtant des indices de traitements récents. S'il n'est pas aisé de savoir si les parcelles, qui doivent être analysées, viennent ou non d'être traitées en l'absence du viticulteur concerné, l'odeur demeure - malgré son manque de fiabilité car certains pesticides sont inodores - l'indicateur majeur d'une intervention récente.

«Dans une parcelle qui sentait où on s'était arrêté, on n'allait pas au milieu de la vigne, mais on allait faire quelques observations. Mais là, moi je m'en vais, je remonte dans la voiture, mais avant, non. Mais c'est pas parce que ça sentait mauvais que c'était forcément plus toxique. Mais il y avait l'indicateur : elle a été traitée récemment. »

(Technicien CA, Hérault)

Cependant, face aux situations à risque, leurs réponses ne sont pas homogènes. Tandis que les hommes cherchent à ne pas intervenir lorsqu'ils risquent d'être exposés à des traitements récents, les femmes refusent d'intervenir dans de telles situations. Leur refus s'inscrit dans la perspective du droit de retrait, qui permet au salarié de se soustraire à « un danger grave et imminent pour sa vie ou sa santé » (art. L. 4131-1 C. trav.). Plus particulièrement pour les femmes enceintes, la référence est celle du droit québécois. Là, en s'appuyant sur la «loi sur la santé et la sécurité du travail », « environ $40 \%$ des travailleuses enceintes font une demande d'évaluation 
des risques présents dans leur milieu de travail » (Denhez, 2003). En s'appropriant implicitement ces textes, qui autorisent le retrait des situations d'exposition à des toxiques, et en agissant de manière informelle mais collective, les techniciennes interpellent aussi bien leurs collègues masculins que leur hiérarchie et les autorités sanitaires. En initiant une dynamique du retrait des situations d'exposition, elles s'inscrivent dans une histoire sociale qui cherche à faire reconnaître les risques des métiers (Cassou et al., 1985). En s'appuyant aussi sur l'importance de la maternité, elles reprennent un questionnement qui a organisé les préoccupations de santé au travail des femmes au cours du $\mathrm{XX}^{\mathrm{e}}$ siècle (Fellinger, 2008).

"Quand on a eu trois jours de récupération de produits phyto non utilisables... On a eu deux, trois collègues femmes qui n'ont pas voulu y aller. Personne n'a pu les obliger à y aller... Et ça a démarré chez elles. Il y a eu une journée de formation. Mais on a une collègue viti qui a refusé d'y aller, on lui a dit: "Mais quand tu fais tes tournées, que tu as le nez dedans, le gars s'il a traité la veille avec un produit qui sent pas, tu vas en prendre encore plus." Elle dit : "Je sais, mais là je peux pas m'y faire.” "

(Technicien CA, Hérault)

"On a une collègue qui est enceinte, elle va pas aller dans les vignes jusqu'à ce qu'elle s'en aille, alors qu'il y a 4 ou 5 ans... »

(Technicien CA, Hérault)

Les enquêtes européennes sur les conditions de travail montrent que les hommes ont tendance à sous-estimer l'impact de conditions de travail qui relèveraient de la masculinité, telles que l'exposition à des toxiques, alors que les femmes y seraient plus attentives (Gollac et Volkoff, 2006). Ainsi, en n'étant pas une situation de travail ordinairement féminine, l'exposition aux pesticides serait d'autant plus conçue comme une situation anormale de travail pour elles. Ce clivage parmi les techniciens met aussi paradoxalement en avant leur culture commune de salariés. Cette culture s'adosse à l'acceptation de l'éventuel port d'attributs du risque que sont les équipements de protection mis à disposition par l'employeur. Mais une telle reconnaissance du risque n'est pas accompagnée, comme dans les autres secteurs impliqués (chimie...), de ses attributs complémentaires habituels (procédures, prime de risque, visite médicale spécifique...). S’il n'est pas certain qu'ils veuillent se voir attribuer ces compléments, la posture de retrait des techniciens peut être vue, à tout le moins, comme une quête de reconnaissance publique du risque pris, autant par les viticulteurs pour qui ils effectuent les prélèvements, que par leur hiérarchie. C'est aussi, en direction de la médecine du travail $1^{5}$, une quête de reconnaissance de l'origine professionnelle des maux subis.

De même que les viticulteurs, les techniciens, en prenant des risques accrus, font montre d'altruisme, en effectuant certaines activités des techniciennes pour les protéger ; la prise de risque se fait au nom d'un intérêt supérieur : celui du collectif de travail et de ses partenaires. En ce sens, elle s'intègre dans une éthique du métier.

\section{Deux cultures professionnelles du risque qui conduisent à une appropriation distincte ?}

L'évolution des comportements des techniciens et conseillers viticoles est significative d'une prise de conscience des risques impliqués par l'usage des pesticides en viticulture. Il est remarquable que cette initiative provienne des femmes et se poursuive au-delà des situations de grossesse. Ce mouvement de retrait, qui se développe chez les conseillères, souligne

5. Cf. l'analyse de sang de la technicienne, l'affectation des cordes vocales du technicien... 
que la relation aux risques dépend également des rapports sociaux de sexe, car les hommes peuvent être autant affectés dans leur santé reproductive (Tron et al., 2001). Cependant, contrairement aux viticulteurs, les conseillers débattent des maux qu'ils ressentent. Au travers de ces échanges, ces maux prennent le statut de problèmes du travail et deviennent reconnus comme tels, au moins parmi les pairs. La posture des conseillers vise à montrer que la reconnaissance collective des risques liés à leur exposition aux pesticides, qui émerge ainsi, devient un problème du métier. Dans l'exercice de ce métier, leurs pratiques prudentielles mettent en évidence des accords collectifs qui tranchent avec la relégation dans le domaine privé des problèmes de santé des viticulteurs. Chez ces derniers, les expériences partagées, même les plus ultimes, ne sont pas débattues collectivement. Elles alimentent des propos privés et cette inscription dans la sphère privée individualise le phénomène, en l'excluant de la sphère professionnelle.

«Le cancer, mon père est mort de ça. Ma mère allait pas à la vigne, mon père fumait un peu, pas beaucoup, il buvait beaucoup de vin comme tous les viticulteurs, je me suis dit: il est mort de ça. Trois ans après, elle est décédée de ça, elle buvait pas, elle fumait pas. C'est qu'il y avait autre chose. Ils avaient un jardin, ils employaient de l'insecticide tout ça, mais peut-être que c'est pas ça. Des fois, on se pose des questions. Moi ça m'a interpellé. J'ai demandé si génétiquement c'était... On m'a dit que c'était pas du tout génétique. Mais enfin bon, quand les deux ont le même... Le professeur disait : c'est très rare. C'est sûrement dû̀ à l'alimentation. »

(Viticulteur, Hérault, $n^{\circ}$ 17)

La sphère privée est cependant un lieu de préservation de la santé des membres de la famille, dont sont chargées les femmes (Saillant, 1991). Les viticulteurs ne font pourtant pas référence aux éventuels soucis et pressions de leurs compagnes. Leur transformation des problèmes de santé au travail en faits privés, confortée par les institutions sanitaires, permet autant de prévenir tout débat que de rassurer celui ou celle qui s'interroge. Dans un monde viticole en restructuration par la rurbanisation et le développement des cahiers des charges qui dissocient les collectifs, les réponses des viticulteurs s'individualisent d'autant plus. D'un autre côté, en considérant que les distinctions culturelles clivent le rapport au corps et à ses usages (Bourdieu, 1979), la virilité des techniciens diffère peu de celle des viticulteurs, lorsqu'elle leur permet de masquer leur peur, au travers de la dimension altruiste de leur prise de risque pour protéger leurs collègues plus vulnérables.

Les voies de leur normativité, structurées par des cultures de métiers différenciées, clivent les rapports à l'exposition aux risques des viticulteurs et des techniciens. Les distinctions qui prennent forme à l'intérieur de chacun des groupes posent la question d'une possible évolution de la normalité sanitaire du travail en viticulture. Au-delà, le cloisonnement des sphères dans lesquelles est interrogée l'exposition de populations à des pesticides et leurs effets sanitaires limitent actuellement les débats.

$$
\begin{gathered}
* \\
* *
\end{gathered}
$$

Dans un contexte de crises viticoles récurrentes en France, gérer leur exposition au risque dans une perspective prudentielle par les viticulteurs est moins aisé encore : se protéger du risque à l'aide d'équipements n'est pas accessible à tous. En effet, les inégalités socio-économiques pèsent sur les choix, tandis que la désagrégation de l'organisation sociale que provoquent les crises renforce l'individualisation des réponses aux questions posées (Castel, 1995). D'un côté, elle suscite l'interrogation des individus face aux risques; d'un autre, elle les contraint à accepter des 
conditions de travail dégradées. Mais la crise peut aussi être, paradoxalement, une opportunité pour ces travailleurs indépendants soucieux d'économies. C'est un tel souci qui a guidé les précurseurs de la Région qui ont élaboré un cahier des charges de réduction d'usage des pesticides.

"Au départ, on avait monté un petit groupe de comité raisonné, mais c'était surtout pour faire des économies... Donc on est parti en Espagne chercher des produits... Et puis on a dit : maintenant il faut aller un peu plus loin, il faut essayer de traiter moins et l'économique passera par là. Au départ, on était quatre, je crois... on a travaillé sur le mildiou, ce qui était le plus simple... On arrivait certaines années à ne pas traiter le mildiou... Le premier point, c'était de l'économique. »

(Viticulteur, réunion coopérative, Aude)

Dès lors, la normativité au travail des viticulteurs comme des techniciens s'exprime en des pratiques prudentielles, pour limiter leur exposition aux pesticides. Ce faisant, ces pratiques interpellent autant les bonnes manières de faire que les normalités sanitaires établies. Aujourd'hui, les clivages semblent plus manifestes que les convergences. C'est aussi parce que les pratiques d'usage des pesticides demeurent socialement pertinentes pour relier les différentes catégories de viticulteurs au sein de leurs communautés de pratiques territorialisées. Tandis que les pratiques prudentielles s'inscrivent dans une remise en cause des collectifs mythiques de la population des coopérateurs. C'est donc à partir des expériences de réduction de l'usage des pesticides prenant en compte la diversité des populations au travail - ainsi du parrainage mis en place par une coopérative de l'Aude - comme des pratiques prudentielles élaborées à bas bruit par des viticulteurs ou des techniciens agricoles, et parfois ensemble, que pourrait évoluer la normalité sanitaire du travail des viticulteurs et des techniciens viticoles.

\section{RÉFÉRENCES BIBLIOGRAPHIQUES}

Bourdieu P. (1979). La distinction, critique sociale du jugement. Paris, Éditions de Minuit.

Brives H. (2006). Les conseillers agricoles et l'environnement: quelles compétences? In J. Rémy, H. Brives, B. Léméry (dir.), Conseiller en agriculture (p. 169-182). INRA-Educagri.

Cadé M. (1994). Traditions identitaires du mouvement ouvrier français dans le Midi rouge, de la fin du XIX ${ }^{\mathrm{e}}$ siècle à nos jours. Le Mouvement Social, ${ }^{\circ}$ 166, p. 91-105.

Canguilhem G. (1966). Le normal et le pathologique. Paris, Presses universitaires de France, 225 p.

Organisation et intensité du travail. Toulouse, Octarès, p. 335-343.

Cassou B., Huez D., Mousel M. L., Spitzer C., Touranchet A. (1985). Les risques du travail.
Pour ne pas perdre sa vie à la gagner. Paris, La Découverte, $640 \mathrm{p}$.

Castel R. (1995). Les métamorphoses de la question sociale. Paris, Fayard, 490 p.

Compagnone C., Cerf M. (2000). Utiliser et engendrer des communications pour accompagner le changement des compétences: le cas du conseil en agriculture. Actes du $35^{\mathrm{e}}$ Congrès de la SELF, p. 354-362.

Compagnone C. (2006). Le juste dans la relation de conseil en agriculture. In J. Rémy, H. Brives, B. Léméry (dir.), Conseiller en agriculture (p. 221-234). INRA-Educagri.

Cru D., Dejours C. (1983). Les savoir-faire de prudence dans les métiers du bâtiment. Les cahiers médicaux-sociaux, $\mathrm{n}^{\circ} 3$, p. 239-247.

De Gasparo S., Dejours C., Nicourt C. (2006). Interpellations environnementales d'éle- 
RECHERCHES

vages et vécu au travail d'éleveurs: une contribution à l'évolution d'un métier ? In P. Askenazy, D. Cartron, F. de Coninck, M. Gollac (dir.). Organisation et intensité du travail (p. 335-343). Toulouse, Octarès.

Dejours C. (2000). Travail, usure mentale. Paris, Bayard, $3^{\mathrm{e}}$ éd., 281 p.

Denhez L. (2003). Mise à jour de l'analyse de risque humaine en regard de l'épandage de pesticides pour la travailleuse enceinte ou qui allaite. Institut National de Santé Publique du Québec, 11 p.

Fellinger A. (2008). Du soupçon à la radioprotection: les scientifiques face au risque professionnel de la radioactivité en France (1901-1967). Thèse, Université de Strasbourg.

Garrigou A. (2007). Une approche ergotoxicologique de l'efficacité des EPI : le cas de l'usage des produits phytosanitaires en viticulture, SFRP. Bordeaux, http://www.sfrp. asso.fr/MAN/pdf/19-Garrigou.pdf.

Garrigou A., Baldi I., Dubuc P. (2008). Apports de l'ergotoxicologie à l'évaluation de l'efficacité réelle des EPI devant protéger $d u$ risque phytosanitaire: de l'analyse de la contamination au processus collectif d'alerte. Pistes, 10, 1, 17 p.

Gollac M., Volkoff S. (2006). La santé au travail et ses masques. Actes de la recherche en sciences sociales, vol. $3, \mathrm{n}^{\circ} 163, \mathrm{p} .4-17$.

Hughes E. C. (1996). Le regard sociologique, essais choisis. Paris, EHESS. 344 p.

Jollivet M., Mathieu N. (1989). Du rural à l'environnement: la question de la nature aujourd'hui. Paris, L'Harmattan, 351 p.
Linhart D. (2009). Travailler sans les autres. Paris, Seuil, 213 p.

Loriol M. (2000). Le temps de la fatigue: la gestion du mal-être au travail. Paris, Anthropos. 293 p.

Luginbühl Y. (1991). Le paysage rural. La couleur de l'agricole, la saveur de l'agricole, mais que reste-t-il de l'agricole? Études Rurales, $\mathrm{n}^{\circ} 121-124$, p. 27- 44.

Moriceau C. (2009). Les perceptions des risques au travail dans la seconde moitié du XIX ${ }^{\mathrm{e}}$ siècle: entre connaissance, déni et prévention. Revue d'histoire moderne et contemporaine, vol. 56, $\mathrm{n}^{\circ}$ 1, p. 11-27.

Nicourt C., Garcia-Parpet M.-F, Girault J.-M., Ollivier D. (2007). De nouvelles pratiques réduisant les risques phytosanitaires: un enjeu dans la construction de la qualité $d u$ vin. Rapport, Ivry, INRA, $121 \mathrm{p}$.

Saillant F. (1991). Les soins en péril : entre la nécessité et l'exclusion. Recherches féministes, vol. 4, $\mathrm{n}^{\circ} 1$, p. 11-29.

Tardieu A. (1862). Dictionnaire d'hygiène publique et de salubrité. Paris, Baillière, 3 tomes, $560 \mathrm{p}$.

Trompette P., Caroly S. (2004). En aparté avec les morts... Peurs, larmes et rire au travail : les métiers du funéraire. Terrain, $\mathrm{n}^{\circ} 43$, p. 63-84.

Tron I., Piquet O., Cohuet S. (2001). Effets chroniques des pesticides sur la santé : état actuel des connaissances. Rennes, Observatoire Régional de la Santé Bretagne, 88 p.

Wenger E. (1998). Communities of practice: learning, meaning, and identity. New York, Cambridge University Press, 340 p. 